\title{
Flows induced by inclined rotation in directional solidification of variable-viscosity solutions
}

\author{
Min Hsing Chang ${ }^{\mathrm{a}}$, Falin Chen ${ }^{\mathrm{b}, *}$ \\ ${ }^{a}$ Department of Mechanical Engineering, Yung-Ta College of Technology, Pingtung, Taiwan 909, ROC \\ ${ }^{\mathrm{b}}$ Institute of Applied Mechanics, National Taiwan University, Taipei, 10764 Taiwan 106, ROC
}

Accepted 8 August 2001

Communicated by T. Nishinaga

\begin{abstract}
As the unidirectional-solidifying melt rotates with respect to an inclined axis, a shear flow is induced to move parallel to the melt/solid interface while it changes its direction along the axis perpendicular to the interface, like an Ekman spiral flow. This induced flow will in turn alter the morphology of the solidifying melt/solid interface and eventually influence the quality of the final casting. In the present paper, we investigate this induced flow in the system rotating in a general way while physical conditions vary: different rotating conditions, different thermal and solutal gradients, and the non-Boussinesq effect due to variable viscosity of the melt. Results show that, in brief, the induced flow is driven by the gravity due to inclination while modified by rotation. As each of the following parameters is higher: the effective Taylor number $\left(T_{\mathrm{e}}\right)$, the thermal Rayleigh number $\left(R_{\mathrm{t}}\right)$, the solutal Rayleigh number $\left(R_{\mathrm{c}}\right)$, or the Lewis number $(\mathrm{Le})$, the induced flow is of larger velocity. The variable-viscosity effect is more complex, depending on the value of other physical parameters. (C) 2001 Elsevier Science B.V. All rights reserved.
\end{abstract}

Keywords: A1. Convection; A1. Directional solidification; A1. Fluid flows; A1. Morphological stability; A2. Growth from melt; A2. Industrial crystallization

\section{Introduction}

The directional solidification is one of the major industrial techniques by which semiconductor materials such as silicon and gallium-arsenic are manufactured. During the solidification process, the latent heat is released from the melt/solid interface due to phase change and is conducted away into the solid and the melt, forming a thermal boundary layer above the interface. In the

\footnotetext{
*Corresponding author. Fax: + 886-2-3639290.

E-mail address: falin@iam.ntu.edu.tw (F. Chen).
}

meantime, a compositional boundary layer forms above the interface because the solidifying binary solution rejects or incorporates the solute at the interface, enhancing the solutal diffusion in the vicinity of the melt/solid interface. After the thermal and compositional boundary layers form, the morphological instability may occur and disturb the interface into non-planar shape [1]. On the other hand, another kind of instability, or the convective instability, can also occur if the rejected solute is lighter or the incorporated solute is heavier than the solvent, rendering the density distribution in the compositional boundary layer 
hydro-statically unstable and eventually leading to the onset of double-diffusive convection above the interface [2]. This convective instability may couple with the morphological instability and result in an undesired change of the quality of final casting. An effective scheme to control these two instability modes has accordingly become an important issue discussed in the research of directional solidification, and meanwhile the imposition of a shear flow in the melt of directionally solidifying solution has been shown to be a potentially effective scheme to control these stability modes as well as to enhance the quality of the final casting.

The influence of the imposed shear flow on these two instability modes was first investigated by Delves $[3,4]$ who imposed quadratic and Blasius boundary-layer flows and by Coriell et al. [5] who imposed a plane Couette flow onto the melt/solid interface. Later, MacFadden et al. [6] imposed a plane stagnation flow vertically onto the melt/solid interface and Forth and Wheeler [7,8] imposed an asymptotic suction boundary-layer flow above the interface. All these studies concluded that the imposed shear flow inhibits the instability modes propagating in a direction being non-perpendicular to the imposed flow (the modes containing transverse components). Recently, however, Chung and Chen [9] showed that as the imposed shear flow (a Blasius type boundary-layer flow) becomes strong enough, a transverse mode (rollaxis perpendicular to the imposed flow) of very unstable characteristics is induced. This mode could exist even without buoyancy. Instead of imposing the shear flow artificially as did by the above-mentioned studies, Sample and Hellawell [10] proposed to rotate the casting mode with respect to an axis inclined to the gravity so that a shear flow can be induced naturally due to inclination. Although no vigorous evidence was provided, they nevertheless conjectured that the shear flow might inhibit greatly the instability of the melt solidifying from below. Their conjecture was later investigated analytically by Chung and Chen [11], in which they showed that a strong helical shear flow was induced and the shear flow generally enhanced the stability of the system.
Since the inclined rotation generates a naturally induced shear flow, instead of an artificial imposed flow as did by previous studies, it is therefore more feasible for the industrial purpose. We accordingly investigate in the present paper in a broad physical sense to study the change of the induced flow under various physical situations. Special emphasis is placed on both the effects due to varying physical parameters and the non-Boussinesq effect due to temperature-dependent viscosity because the viscosity of the melt may change significantly during solidification due to temperature variation. For example, the viscosity of glass forming alloys decreases by $O\left(10^{20}\right)$ when temperature decreases from working condition to melting point [12] and, for lead-tin alloy, it changes by a factor of two when temperature changes over $200 \mathrm{~K}$ [13]. Moreover, since the temperature-dependent viscosity changes dramatically in the region above the melt/ solid interface because of the sharp temperature variation, it is expected that the temperaturedependent viscosity may also influence significantly on the induced shear flow, which is largely confined to the region above the interface.

In the present paper, we consider the directional solidification of binary alloy under inclined rotation, in which a shear flow is possibly induced by inclination and modified by rotation as well as the viscosity variation. We present the mathematical model of the flow induced by the inclined rotation in Section 2. The equations are solved by an analytical approach and the solutions are shown in Section 3. Based on this solution, the features of the induced flow are discussed in Section 4. The changes of the induced flow under different physical situations including variable viscosity are discussed in Section 5. Finally, concluding remarks are given in Section 6.

\section{Problem description and formulation}

Consider the system as shown in Fig. 1, a dilute binary solution of temperature $T_{\infty}$ and concentration $C_{\infty}$ is solidifying upwards, in which a solid layer is formed below the semi-infinite bulk melt. The melt/solid interface described by $z=h(x, y, t)$ is assumed to be initially planar and advances into 


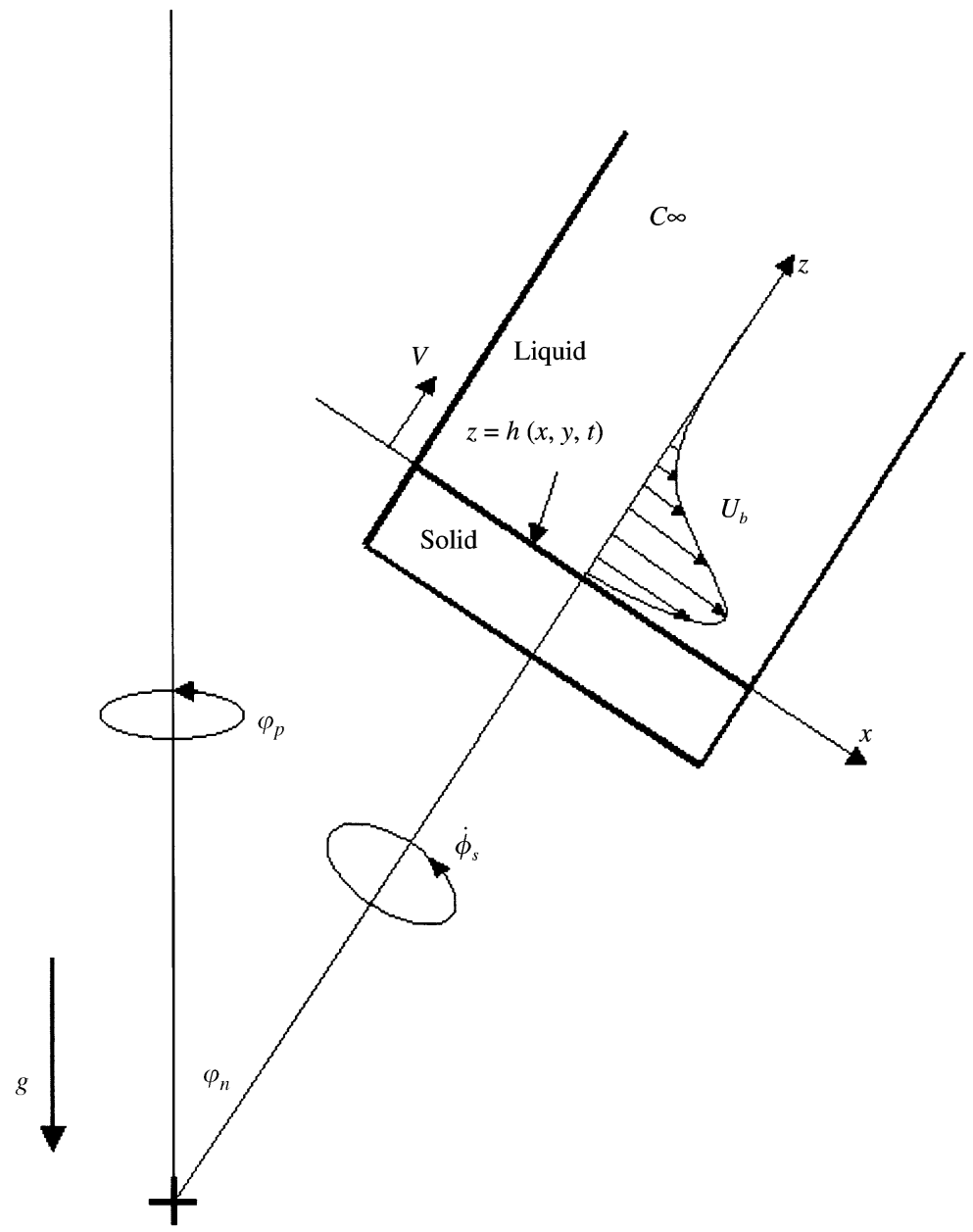

Fig. 1. The schematic description of the system considered. The solid layer is solidifying with a constant velocity $V$ upwards. An induced flow moves parallell along the melt/solid interface. The casting mode rotates, including spinning and precession, with respect to an inclined axis.

the fluid with a constant speed $V$. The cooling tank rotates in a general way including precession and spin that the angular velocity can be expressed by

$$
\begin{aligned}
\dot{\Phi}= & \left(\dot{\phi}_{\mathrm{p}} \sin \phi_{\mathrm{n}} \sin \phi_{\mathrm{s}}\right) \mathbf{e}_{x}+\left(\dot{\phi}_{\mathrm{p}} \sin \phi_{\mathrm{n}} \cos \phi_{\mathrm{s}}\right) \mathbf{e}_{y} \\
& +\left(\dot{\phi}_{\mathrm{p}} \cos \phi_{\mathrm{n}}+\dot{\phi}_{\mathrm{s}}\right) \mathbf{e}_{z},
\end{aligned}
$$

where $\phi_{\mathrm{p}}, \phi_{\mathrm{n}}$ and $\phi_{\mathrm{s}}$ are the angles of precession, nutation and spin, respectively and $\dot{\phi}_{\mathrm{p}}$ and $\dot{\phi}_{\mathrm{s}}$ are the angular velocities of precession and spin, respectively (see Fig. 1). Besides, $\mathbf{e}_{x}, \mathbf{e}_{y}$ and $\mathbf{e}_{z}$ are the unit vectors of Cartesian coordinate, which sits on the melt/solid interface as denoted by the $x-y-z$ frame in Fig. 1 .
In such a coordinate system, the governing equations in the fluid region $h<z<\infty$ are

$$
\nabla \cdot \mathbf{u}=0
$$

$$
\begin{aligned}
& \left(\frac{\partial}{\partial t}-V \frac{\partial}{\partial z}+\mathbf{u} \cdot \nabla\right) \mathbf{u}+2 \dot{\Phi} \times \mathbf{u} \\
& \quad=-\frac{\nabla P}{\rho_{0}}+\left(\frac{\rho}{\rho_{0}}-1\right) \mathbf{g}+\frac{1}{\rho_{0}} \nabla \cdot(\mu \mathbf{D}),
\end{aligned}
$$

$$
\left(\frac{\partial}{\partial t}-V \frac{\partial}{\partial z}+\mathbf{u} \cdot \nabla\right) C=D_{\mathrm{f}} \nabla^{2} C
$$




$$
\left(\frac{\partial}{\partial t}-V \frac{\partial}{\partial z}+\mathbf{u} \cdot \nabla\right) T=k_{\mathrm{f}} \nabla^{2} T
$$

In above equations, $\mathbf{u}$ represents the velocity vector $(u, v, w)$ measured with respect to the cooling tank, $P$ is the pressure, and $\rho_{0}$ is the reference density. The density of the fluid is assumed to be constant except in the gravity term that $\rho=\rho_{0}\left(1-\alpha\left(T-T_{\mathrm{m}}\right)-\beta C\right)$, where $\alpha$ and $\beta$ are the thermal and solute expansion coefficients, respectively, and $T_{\mathrm{m}}$ represents the freezing temperature of the pure solvent. The vector of gravitational acceleration $\mathbf{g}=-g\left(\sin \phi_{\mathrm{n}} \sin \phi_{\mathrm{s}} \mathbf{e}_{x}+\right.$ $\sin \phi_{\mathrm{n}} \cos \phi_{\mathrm{s}} \mathbf{e}_{y}+\cos \phi_{\mathrm{n}} \mathbf{e}_{z}$ ) depends on both the nutation and spin angles, where $g$ is the gravitational constant. $\mathbf{D}=\nabla \mathbf{u}+\nabla \mathbf{u}^{\mathrm{T}}$ is the deviatoric strain tensor and the superscript " $T$ " denotes the transverse of the tensor, $C$ is the concentration, $D_{\mathrm{f}}$ is the solute diffusivity, $T$ is the temperature and $k_{\mathrm{f}}$ is the thermal diffusivity. The viscosity of the fluid $\mu$ is considered as an exponential function of temperature

$\mu=\mu_{0} \exp \left[-c\left(T-T_{\mathrm{m}}\right)\right]$,

where $c>0$ is an arbitrary constant, indicating that the viscosity of the fluid decreases with increasing temperature, and $\mu_{0}=v_{0} \rho_{0}$ is the reference dynamic viscosity, $v_{0}$ is the reference kinematic viscosity. The exponential model is applied because of its wider use for hydrogenbonded liquids than the other models [14], which suits the aqueous ammonium chloride solution considered in the present paper well.

In the solid region $z<h$, we neglect the diffusion of solute concentration and consider the diffusion of heat only. Then the heat equation is

$$
\left(\frac{\partial}{\partial t}-V \frac{\partial}{\partial z}\right) T=k_{\mathrm{s}} \nabla^{2} T
$$

where $k_{\mathrm{s}}$ is the thermal diffusivity of the solid phase. The boundary conditions at infinite far field are assumed to be that the fluid experiences a rigidbody rotation with the tank and both the concentration and temperature of the fluid remain unchanged during solidification process. Furthermore, it is assumed that the height of the tank is large enough that the influence on the induced flow due to the possible deformation of the free surface can be neglected. Accordingly, at the infinite far field $z \rightarrow \infty$ we have

$\mathbf{u} \rightarrow 0, \quad C \rightarrow C_{\infty}, \quad T \rightarrow T_{\infty}$.

At the melt/solid interface $z=h(x, y, t)$, the boundary conditions are

$$
\begin{aligned}
& \mathbf{u} \times \mathbf{n}=0, \\
& \mathbf{u} \cdot \mathbf{n}=0, \\
& C^{+}(1-k)\left(V+\frac{\partial h}{\partial t}\right) \mathbf{e}_{z} \cdot \mathbf{n}=-D_{\mathrm{f}} \frac{\partial C^{+}}{\partial n}, \\
& T^{+}=m C^{+}+T_{\mathrm{m}}(1-\Gamma \xi), \\
& T^{+}=T^{-}, \\
& L\left(V+\frac{\partial h}{\partial t}\right) \mathbf{e}_{z} \cdot \mathbf{n}=\kappa_{\mathrm{s}} \frac{\partial T^{-}}{\partial n}-\kappa_{\mathrm{f}} \frac{\partial T^{+}}{\partial n},
\end{aligned}
$$

where $\mathbf{n}$ is the unit vector normal to the interface directing toward the fluid. The superscripts "+" and "-" account for the quantities right above and below the interface, respectively. Eq. (2.6a) is the no-slip condition. Eq. (2.6b) expresses the conservation of mass at the interface by neglecting the difference of the densities between the solid and liquid phases. Eq. (2.6c) accounts for the conservation of the solute across the interface, where $k=C^{-} / C^{+}$is the segregation or partition coefficient. Eq. (2.6d) is the thermal-dynamical equilibrium condition that describes the dependence of the freezing temperature of a binary alloy upon its composition. The capillary effect (the Gibbs-Thompson effect) is included in the last term and the liquidus slope $m$ is assumed to be a constant. $\Gamma$ is the capillary length and $\xi$ is the curvature of the interface that is assumed to be negative for a concave projection into the fluid. Eq. (2.6e) expresses the continuity of the temperature across the interface and Eq. (2.6f) accounts for the energy balance at the interface, where $\kappa_{\mathrm{s}}$ and $\kappa_{\mathrm{f}}$ are the thermal conductivity of the solid and fluid, respectively, $L$ is the latent heat per unit volume of solid.

The governing equations as well as the boundary conditions are non-dimensionalized with the following solute-field scales: $V$ for velocity, $H=D_{\mathrm{f}} / V$ for length, $D_{\mathrm{f}} / V^{2}$ for time, $C_{\infty}$ for 
concentration, $T_{\mathrm{m}}$ for temperature and $v_{0} \rho_{0} V^{2} / D_{\mathrm{f}}$ for pressure. Note that the temperature is subtracted by $T_{\mathrm{m}}$ before dividing it by the scale. Then the dimensionless viscosity function $\mu$ can be written as

$\mu=\exp \left[\gamma\left(-T / T_{\infty}\right)\right]$,

where $\gamma=\ln \left(\mu_{0} / \mu_{\infty}\right)$ accounts for the viscosity contrast between the fluid at the freezing temperature and the bulk fluid at infinity, $T$ is the dimensionless temperature and $T_{\infty}$ is the dimensionless temperature at infinity. As a result, the dimensionless governing equations in the fluid are

$\nabla \cdot \mathbf{u}=0$

$$
\begin{aligned}
\mathrm{Sc}^{-1} & \left(\frac{\partial}{\partial t}-\frac{\partial}{\partial z}+\mathbf{u} \cdot \nabla\right) u \\
= & -\frac{\partial P}{\partial x}+\left(R_{\mathrm{c}} C+R_{\mathrm{t}} T\right) S_{\mathrm{n}} S_{\mathrm{s}}(t) \\
& +(\nabla \cdot \mu \mathbf{D}) \cdot \mathbf{e}_{x} \\
& +\left[(-1)^{n_{\mathrm{p}}} \mathrm{Ta}_{\mathrm{p}}^{1 / 2} C_{\mathrm{n}}+(-1)^{n_{\mathrm{s}}} \mathrm{Ta}_{\mathrm{s}}^{1 / 2}\right] v \\
& -\left[(-1)^{n_{\mathrm{p}}} \mathrm{Ta}_{\mathrm{p}}^{1 / 2} S_{\mathrm{n}} C_{\mathrm{s}}(t)\right] w,
\end{aligned}
$$

$$
\begin{aligned}
\mathrm{Sc}^{-1} & \left(\frac{\partial}{\partial t}-\frac{\partial}{\partial z}+\mathbf{u} \cdot \nabla\right) v \\
= & -\frac{\partial P}{\partial y}+\left(R_{\mathrm{c}} C+R_{\mathrm{t}} T\right) S_{\mathrm{n}} C_{\mathrm{s}}(t)+(\nabla \cdot \mu \mathbf{D}) \cdot \mathbf{e}_{y} \\
& +\left[(-1)^{n_{\mathrm{p}}} \mathrm{Ta}_{\mathrm{p}}^{1 / 2} C_{\mathrm{n}}+(-1)^{n_{\mathrm{s}}} \mathrm{Ta}_{\mathrm{s}}^{1 / 2}\right] u \\
& +\left[(-1)^{n_{\mathrm{p}}} \mathrm{Ta}_{\mathrm{p}}^{1 / 2} S_{\mathrm{n}} S_{\mathrm{s}}(t)\right] w
\end{aligned}
$$

$$
\begin{aligned}
\mathrm{Sc}^{-1} & \left(\frac{\partial}{\partial t}-\frac{\partial}{\partial z}+\mathbf{u} \cdot \nabla\right) w \\
= & -\frac{\partial P}{\partial z}+\left(R_{\mathrm{c}} C+R_{\mathrm{t}} T\right) C_{\mathrm{n}} \\
& +(\nabla \cdot \mu \mathbf{D}) \cdot \mathbf{e}_{z}+\left[(-1)^{n_{\mathrm{p}}} \mathrm{Ta}_{\mathrm{p}}^{1 / 2} S_{\mathrm{n}} C_{\mathrm{s}}(t)\right] u \\
& -\left[(-1)^{n_{\mathrm{p}}} \mathrm{Ta}_{\mathrm{p}}^{1 / 2} S_{\mathrm{n}} S_{\mathrm{s}}(t)\right] v,
\end{aligned}
$$$$
\left(\frac{\partial}{\partial t}-\frac{\partial}{\partial z}+\mathbf{u} \cdot \nabla\right) C=\nabla^{2} C
$$$$
\mathrm{Le}^{-1}\left(\frac{\partial}{\partial t}-\frac{\partial}{\partial z}+\mathbf{u} \cdot \nabla\right) T=\nabla^{2} T,
$$

and the dimensionless heat balance equation in the solid layer is

$L s^{-1}\left(\frac{\partial}{\partial t}-\frac{\partial}{\partial z}\right) T=\nabla^{2} T$.

In above equation, $\mathrm{Sc}=v_{0} / D_{\mathrm{f}}$ is the Schmidt number, Le $=k_{\mathrm{f}} / D_{\mathrm{f}}$ is the Lewis number of fluid and $L s^{-1}=k_{\mathrm{s}} / D_{\mathrm{f}}$ is the Lewis number of solid, $R_{\mathrm{c}}$ and $R_{\mathrm{t}}$ are the solute and thermal Rayleigh numbers, respectively defined by

$R_{\mathrm{c}}=\frac{g \beta C_{\infty} H^{3}}{v_{0} D_{\mathrm{f}}}, \quad R_{\mathrm{t}}=\frac{g \alpha T_{\mathrm{m}} H^{3}}{v_{0} D_{\mathrm{f}}}$.

The parameters $\mathrm{Ta}_{\mathrm{p}}$ and $\mathrm{Ta}_{\mathrm{s}}$ are the Taylor numbers of precession and spin, respectively, and are defined by

$\mathrm{Ta}_{\mathrm{p}}=\left(\frac{2 H^{2} \dot{\phi}_{\mathrm{p}}}{v_{0}}\right)^{2}, \quad \mathrm{Ta}_{\mathrm{s}}=\left(\frac{2 H^{2} \dot{\phi}_{\mathrm{s}}}{v_{0}}\right)^{2}$.

Some abbreviations have been used and their definitions are shown in the following:

$$
\begin{aligned}
& S_{\mathrm{n}}=\sin \phi_{\mathrm{n}}, \quad C_{\mathrm{n}}=\cos \phi_{\mathrm{n}}, \quad S_{\mathrm{s}}(t)=\sin (\Omega t), \\
& C_{\mathrm{s}}(t)=\cos (\Omega t),
\end{aligned}
$$

where $\Omega=\mathrm{Sc}(-1)^{n_{\mathrm{s}}} \mathrm{Ta}_{\mathrm{s}}^{1 / 2} / 2$ is the dimensionless angular velocity of spin. Note that the exponents $n_{\mathrm{p}}$ and $n_{\mathrm{s}}$ indicate the sense of precession and spin, respectively: As $\dot{\phi}_{\mathrm{p}}>0$ or $\dot{\phi}_{\mathrm{s}}>0$, one obtains $n_{\mathrm{p}}=$ 0 or $n_{\mathrm{s}}=0$, respectively; as $\dot{\phi}_{\mathrm{p}}<0$ or $\dot{\phi}_{\mathrm{s}}<0$, it yields $n_{\mathrm{p}}=1$ or $n_{\mathrm{s}}=1$, respectively.

The dimensionless boundary conditions at $z \rightarrow \infty$ are

$\mathbf{u}=0, \quad C=1, \quad T=T_{\infty}$,

at the melt/solid interface $z=h(x, y, t)$ are

$\mathbf{u} \times \mathbf{n}=0$,

$\mathbf{u} \cdot \mathbf{n}=0$,

$C^{+}(k-1)\left(1+\frac{\partial h}{\partial t}\right) \mathbf{e}_{z} \cdot \mathbf{n}=\nabla C^{+} \cdot \mathbf{n}$,

$T^{+}=M C^{+}-\eta \xi$,

$T^{+}=T^{-}$,

$\mathscr{L}\left(1+\frac{\partial h}{\partial t}\right) \mathbf{e}_{z} \cdot \mathbf{n}=\left(L s^{-1} \nabla T^{-}-\operatorname{Le} \nabla T^{+}\right) \cdot \mathbf{n}$, 
where the parameter $M=m C_{\infty} / T_{\mathrm{m}}$ is the dimensionless liquidus slope, $\eta=\Gamma V / D_{\mathrm{f}}$ is the dimensionless capillary length and $\mathscr{L}=L / T_{\mathrm{m}}\left(\rho C_{\mathrm{p}}\right)^{-}$is the Stephan number. Note that in Eq. (2.14f) the difference of the specific heat per unit volume between the solid and liquid phase is neglected.

\section{Analytical solution of the flow induced by inclination}

The induced flow during solidification before the melt/solid interface losing the planar shape is induced by the inclination of cooling tank while is modified by rotation. To investigate this induced flow analytically, it is assumed that the melt/solid interface remains planar during solidification, namely,

$h_{\mathrm{b}}=0$.

It is assumed also that the horizontal dimension of the cooling tank is much larger than the characteristic length of the solute scale $H$. Consequently, it can be seen from the scale analysis of the continuity equation that the velocity along the height of the tank is much weaker than that along the $x-y$ plane when small-scaled convection is absent from the system, and the differential derivatives in the $x-y$ planes are negligible in comparison with that along the height of tank as the horizontal size of tank is sufficiently large. As a result, the temperature and concentration are not affected by the presence of the flow induced by inclination and then should be similar to those of previous studies $[6,8,15]$, which are shown in the following for convenience for subsequent discussion.

In the melt $z>0$, the concentration and temperature distributions are represented by a concentration boundary layer and a thermal boundary layer, respectively, i.e.,

$C_{\mathrm{b}}=1-G_{\mathrm{c}} \mathrm{e}^{-z}$,

$T_{\mathrm{b}}=T_{\infty}-\mathrm{Le} G_{1} \mathrm{e}^{-z / \mathrm{Le}}$,

where the local concentration gradient and temperature gradient are

$G_{\mathrm{c}}=\frac{k-1}{k}$
$G_{1}=\frac{T_{\infty}-M / k}{\operatorname{Le}}$

In the solid layer $z<0$, the concentration keeps constant and the temperature decreases exponentially,

$C_{\mathrm{b}}=1$,

$T_{\mathrm{b}}=T_{\infty}+\mathscr{L}-\left(\mathscr{L} \mathrm{Le}_{1}\right) \mathrm{e}^{-z / L s}$.

Regarding the induced flow, it is shown from scale analysis that the $z$-component of the induced velocity can be assumed to be zero, i.e.

$w_{\mathrm{b}}=0$.

By applying Eqs. (2.8b)-(2.8d) and boundary condition Equation (2.13), we obtain the pressure distribution in the melt as

$$
\begin{aligned}
P_{\mathrm{b}}= & \bar{P}_{\mathrm{b}}(z, t)+\left(R_{\mathrm{c}}+R_{\mathrm{t}} T_{\infty}\right)\left[x S_{\mathrm{n}} S_{\mathrm{s}}(t)\right. \\
& \left.+y S_{\mathrm{n}} C_{\mathrm{s}}(t)+z C_{\mathrm{n}}\right]
\end{aligned}
$$

The reduced pressure $\bar{P}_{\mathrm{b}}(z, t)$ can be obtained by substituting Eq. (3.9) into Eq. (2.8d) yields

$$
\begin{aligned}
\frac{\partial \bar{P}_{\mathrm{b}}}{\partial z}= & {\left[(-1)^{n_{\mathrm{p}}} \mathrm{Ta}_{\mathrm{p}}^{1 / 2} S_{\mathrm{n}} C_{\mathrm{s}}(t)\right] u_{\mathrm{b}} } \\
& -\left[(-1)^{n_{\mathrm{p}}} \mathrm{Ta}_{\mathrm{p}}^{1 / 2} S_{\mathrm{n}} S_{\mathrm{s}}(t)\right] v_{\mathrm{b}} \\
& +\left[R_{\mathrm{c}}\left(C_{\mathrm{b}}-1\right)+R_{\mathrm{t}}\left(T_{\mathrm{b}}-T_{\infty}\right)\right] C_{\mathrm{n}} .
\end{aligned}
$$

Note that the pressure gradient in $z$-direction balances the $z$-component of the fluid weight, leading the melt far above the solidifying front to be quiescent and makes the whole melt motionless in the $z$-direction. Namely, in the far field from the interface, the pressure gradient can be seen as the buoyancy due to the density variation in the $z$-direction. On the other hand, however, due to the fact that the density changes greatly within both of concentration and thermal boundary layers, the pressure gradient in both $x$ - and $y$-directions cannot balance the weight of the fluid along corresponding directions and causes a flow along the interface, which is then modified by the Coriolis force due to the rotation. The $x$ - and $y$-components $u_{\mathrm{b}}$ and $v_{\mathrm{b}}$ of the induced flow can be solved by substituting Eqs. (3.8) and (3.10) into Eqs. (2.8b) 
and $(2.8 \mathrm{c})$, yielding

$$
\begin{aligned}
& \mu \frac{\mathrm{d}^{2} \hat{U}_{\mathrm{b}}}{\mathrm{d} z^{2}}+\left(\mu^{\prime}+\mathrm{Sc}^{-1}\right) \frac{\mathrm{d} \hat{U}_{\mathrm{b}}}{\mathrm{d} z}-i T_{\mathrm{e}} \hat{U}_{\mathrm{b}} \\
& \quad=\left[R_{\mathrm{c}}\left(C_{\mathrm{b}}-1\right)+R_{\mathrm{t}}\left(T_{\mathrm{b}}-T_{\infty}\right)\right] S_{\mathrm{n}},
\end{aligned}
$$

where $U_{\mathrm{b}} \equiv u_{\mathrm{b}}+i v_{\mathrm{b}}=\hat{U}_{\mathrm{b}} \mathrm{e}^{i \phi_{\mathrm{g}}}, \phi_{\mathrm{g}}=-(\Omega t+\pi / 2)$, $\mu^{\prime}=\mathrm{d} \mu / \mathrm{d} z$ and $T_{\mathrm{e}}$ is defined as

$T_{\mathrm{e}}=(-1)^{n_{p}} \mathrm{Ta}_{\mathrm{p}}^{1 / 2} C_{\mathrm{n}}+\frac{(-1)^{n_{s}}}{2} \mathrm{Ta}_{\mathrm{s}}^{1 / 2}$.

The associated boundary conditions are

$\hat{U}_{\mathrm{b}}=0, \quad$ at $z=0$ and $z \rightarrow \infty$.

Since the gravity component on the $x-y$ plane can be expressed by $g_{x}+i g_{y}=S_{\mathrm{n}} g \mathrm{e}^{i \phi_{\mathrm{g}}}$, where $\phi_{\mathrm{g}}$ indicates the orientation of the gravity component in the $x-y$ plane. Eqs. (3.11) and (3.13) can be solved by asymptotically expanding the dependent variables with respect to $\varepsilon$, where $\varepsilon=1 / \mathrm{Le}$ is usually a small parameter for an alloy,

$\hat{U}_{\mathrm{b}}=\hat{U}_{\mathrm{b} 0}+\varepsilon \hat{U}_{\mathrm{b} 1}+\varepsilon^{2} \hat{U}_{\mathrm{b} 2}+\cdots$,

and the viscosity $\mu$ can be expressed as

$$
\begin{aligned}
\mu= & A \exp [B \exp (-\varepsilon z)] \\
= & Q\left[1-\varepsilon B z+\varepsilon^{2}\left(\frac{B+B^{2}}{2}\right) z^{2}\right. \\
& \left.-\varepsilon^{3}\left(\frac{B+3 B^{2}}{6}\right) z^{3}+\cdots\right],
\end{aligned}
$$

where $A=\exp (-\gamma), B=\gamma \operatorname{Le} G_{1} / T_{\infty}$, and $Q=$ $A \exp B$. By substituting Eqs. (3.14) and (3.15) into Eqs. (3.11) and (3.13) and by equating the same order terms, we obtain the following perturbation equations of different order of $\varepsilon$

$$
\begin{gathered}
O\left(\varepsilon^{0}\right): \quad Q \frac{\mathrm{d}^{2} \hat{U}_{\mathrm{b} 0}}{\mathrm{~d} z^{2}}+\mathrm{Sc}^{-1} \frac{\mathrm{d} \hat{U}_{\mathrm{b} 0}}{\mathrm{~d} z}-i T_{\mathrm{e}} \hat{U}_{\mathrm{b} 0} \\
=\left[R_{\mathrm{c}}\left(C_{\mathrm{b}}-1\right)+R_{\mathrm{t}}\left(T_{\mathrm{b}}-T_{\infty}\right)\right] S_{\mathrm{n}}, \\
O\left(\varepsilon^{1}\right): \quad Q \frac{\mathrm{d}^{2} \hat{U}_{\mathrm{b} 1}}{\mathrm{~d} z^{2}}+\mathrm{Sc}^{-1} \frac{\mathrm{d} \hat{U}_{\mathrm{b} 1}}{\mathrm{~d} z}-i T_{\mathrm{e}} \hat{U}_{\mathrm{b} 1} \\
=Q B\left[z \frac{\mathrm{d}^{2} \hat{U}_{\mathrm{b} 0}}{\mathrm{~d} z^{2}}+\frac{\mathrm{d} \hat{U}_{\mathrm{b} 0}}{\mathrm{~d} z}\right]
\end{gathered}
$$

$$
\begin{aligned}
O\left(\varepsilon^{2}\right): \quad & Q \frac{\mathrm{d}^{2} \hat{U}_{\mathrm{b} 2}}{\mathrm{~d} z^{2}}+\mathrm{Sc}^{-1} \frac{\mathrm{d} \hat{U}_{\mathrm{b} 2}}{\mathrm{~d} z}-i T_{\mathrm{e}} \hat{U}_{\mathrm{b} 2} \\
= & Q B\left[z \frac{\mathrm{d}^{2} \hat{U}_{\mathrm{b} 1}}{\mathrm{~d} z^{2}}+\frac{\mathrm{d} \hat{U}_{\mathrm{b} 1}}{\mathrm{~d} z}\right. \\
& \left.-\frac{(1+B)}{2} z^{2} \frac{\mathrm{d}^{2} \hat{U}_{\mathrm{b} 0}}{\mathrm{~d} z^{2}}-(1+B) z \frac{\mathrm{d} \hat{U}_{\mathrm{b} 0}}{\mathrm{~d} z}\right] .
\end{aligned}
$$

The terms with higher order than $O\left(\varepsilon^{2}\right)$ are neglected. The perturbed boundary conditions are $\hat{U}_{\mathrm{b} 0}=\hat{U}_{\mathrm{b} 1}=\hat{U}_{\mathrm{b} 2}=0 \quad$ at $z=0 \quad$ and $\quad z \rightarrow \infty$.

The solutions of Eqs. (3.16) $\hat{U}_{\mathrm{b} 0}, \hat{U}_{\mathrm{b} 1}$ and $\hat{U}_{\mathrm{b} 2}$ can be obtained by a straight forward derivation, yielding

$$
\begin{aligned}
\hat{U}_{\mathrm{b} 0}= & A_{1} \mathrm{e}^{-z}+A_{2} \mathrm{e}^{-z / \mathrm{Le}}-\left(A_{1}+A_{2}\right) \mathrm{e}^{a z}, \\
\hat{U}_{\mathrm{b} 1}= & \left(B_{1} z+B_{2}\right) \mathrm{e}^{-z}+\left(B_{3} z+B_{4}\right) \mathrm{e}^{-z / \mathrm{Le}} \\
& +\left(B_{5} z^{2}+B_{6} z-B_{2}-B_{4}\right) \mathrm{e}^{a z}, \\
\hat{U}_{\mathrm{b} 2}= & \left(C_{1} z^{2}+C_{2} z+C_{3}\right) \mathrm{e}^{-z} \\
& +\left(C_{4} z^{2}+C_{5} z+C_{6}\right) \mathrm{e}^{-z / \mathrm{Le}} \\
& +\left(C_{7} z^{4}+C_{8} z^{3}+C_{9} z^{2}\right. \\
& \left.+C_{10} z-C_{3}-C_{6}\right) \mathrm{e}^{a z},
\end{aligned}
$$

where the definitions of the coefficients $A_{1}$ and $A_{2}$, $B_{1}-B_{6}$ and $C_{1}-C_{10}$ are shown in the Appendix A, and

$$
\begin{aligned}
& a=-d_{E}^{-1}-\text { if } \\
& d_{\mathrm{E}}=\left[(2 Q \mathrm{Sc})^{-1}+e\right]^{-1}, \\
& e=\left\{\frac{(Q S \mathrm{Sc})^{-2}+\left[(Q \mathrm{Sc})^{-4}+16\left(T_{\mathrm{e}} / Q\right)^{2}\right]^{1 / 2}}{8}\right\}^{1 / 2},
\end{aligned}
$$

$f=$

$$
\frac{T_{\mathrm{e}}}{\left|T_{\mathrm{e}}\right|}\left\{\frac{-(Q \mathrm{Sc})^{-2}+\left[(Q \mathrm{Sc})^{-4}+16\left(T_{\mathrm{e}} / Q\right)^{2}\right]^{1 / 2}}{8}\right\}^{1 / 2} .
$$

The combined effect due to precession and spin on the amplitude of induced velocity are accounted for by the parameter $T_{\mathrm{e}}$ of Eq. (3.12). 


\section{The induced flow}

From Eq. (3.18) one can see, whether the viscosity is constant or variable, the direction of the induced flow varies with time periodically with a frequency equal to the spin angular velocity $\Omega$. The induced velocity varies with the height of the tank and can be separated into three components of different length scales. The first component is of the length scale of the solute boundary layer, varying vertically with the exponential function $\mathrm{e}^{-z}$ and will be called the solutal-layer flow. The second component is of the length scale of the thermal boundary layer, varying with $\mathrm{e}^{-z / \mathrm{Le}}$ and will be called the thermal-layer flow. The third component varies its magnitude with $\mathrm{e}^{-z / d_{\mathrm{E}}}$ and changes its direction with a period $2 \pi / f$, being apparently a kind of spiral Ekman flow and will accordingly be called the Ekmanlayer flow.

To illustrate more clearly the variation of these three components, we show in Fig. 2 the variations
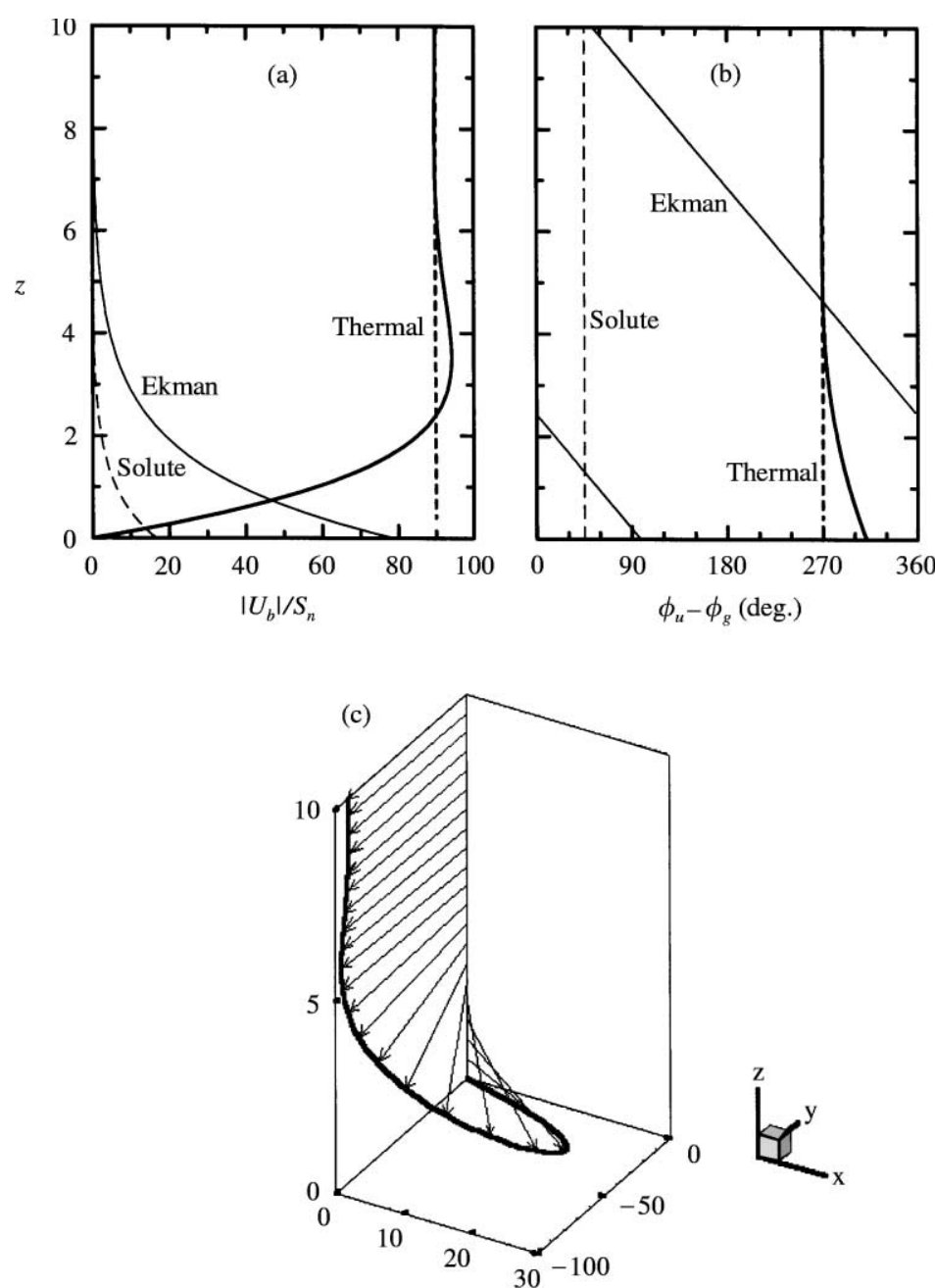

Fig. 2. An example of the induced flow. (a) The velocity amplitudes along the $z$-axis of the three components: the solutal layer flow, the thermal layer flow, and the Ekman layer flow. (b) The phase angle variations of the three flow components; the phase of Ekman layer flow changes periodically with height. (c) The three-dimensional velocity profile of the induced flow, showing that the spiral structure is mainly confined to a shallow layer above the melt/mush interface. 
of both the velocity magnitude and the direction along with the tank height for the case $T_{\mathrm{e}}=1$, $R_{\mathrm{c}}=10, R_{\mathrm{t}}=250, \mathrm{Le}=3600, \mathrm{Sc}=81$, which corresponds to the aqueous ammonium chloride solution of constant viscosity (i.e. $\gamma=0$ ) under small angular velocity rotation (such as $1 \mathrm{rpm}$ ) with an inclined angle about $10^{\circ}$. It is seen from Fig. 2(a) that both the solutal-layer flow and the Ekman-layer flow are of the maximum velocity at the melt/solid interface and decay exponentially with height, while the thermal layer flow is essentially of constant velocity along the height and is of largest velocity compared to the other two components. By combining these three components, the induced flow vanishes at the interface, satisfying the boundary condition, while increases its velocity exponentially with height within the region where both the solutal-layer flow and Ekman-layer flow decay upwards. After reaching the maximum, the induced flow velocity reduces a bit and remains to be that of thermal-layer flow up to the far field. In other words, the induced flow is dominated by the thermal-layer flow in a large region of the melt, while both the solutal-layer flow and the Ekman-layer flow contribute to the induced flow by reducing the thermal-layer flow exponentially to zero when across the shallow region above the interface.

On the other hand, the three component-flows are of different directions, which can be seen from Fig. 2(b) where $\phi_{u}$ is the phase angle of $U_{\mathrm{b}}$. The solutal-layer flow and the thermal-layer flow are of constant directions, differing from the direction of gravity by $45^{\circ}$ and $90^{\circ}$, respectively, while the direction of Ekman-layer flow changes periodically with height, forming the main body of spiral structure. To illustrate the spiral structure, we show the velocity vector in Fig. 2(c) that the induced flow in the far field stays in the $y-z$ plane, while near the interface the flow has a component in $x$-direction. This component has its maximum on the interface and decays rapidly with height. Note that the direction of thermal-layer flow on the interface is virtually opposite to that of Ekman-layer flow, the combination of these two component-flows therefore becomes a flow of small velocity, which is then vanished due to the solutal-layer flow, leading to the non-slip condi- tion on the interface. At $z \approx 3.2$, on the other hand, the Ekman-layer flow and the thermal-layer flow are of the same direction, resulting in the largest amplitude of the induced flow velocity. A small contribution is made by the solutal-layer flow, which modified a little the position of maximum velocity.

The coefficients of the induced flow velocity of Eq. (3.18) contain the parameter $S_{\mathrm{n}}$, indicating that the induced flow is a result of the imbalance between gravity and pressure gradient along $x-y$ plane when the angle between these two forces is larger than zero. On the other hand, one may infer that as the system is rotating with respect to the vertical axis, i.e. the gravity and the pressure gradient are in the same direction, they will have no induced flow irrespective of the fluid viscosity being constant or variable. Because of the inclined rotation, the fluid is driven by the resultant force of gravity and pressure gradient to move parallel along the $x-y$ plane. When the rotation includes spin, the induced flow varies periodically with a frequency $\Omega$, the spin angular velocity, because both the gravity and pressure gradient change their directions with $\Omega$. While this case cannot be applied to the case of precession, since the phase angle difference between the induced flow and the gravity is independent of time, implying that the induced flow due to precession shall be a steady flow. This physical explanation can also apply to the occurrence of the three component-flows: Both the thermal-layer flow and the solutal-layer flow are the results of system inclination while the Ekman-layer flow has mainly resulted from system rotation. In brief, one may conclude that the induced flow is driven by system inclination and is modified by the system rotation.

\section{The physical parameter effects}

In the present system there are several parameters affecting the structure of induced flow. They are the effective Taylor number $T_{\mathrm{e}}$, the solutal Rayleigh number $R_{\mathrm{c}}$, the thermal Rayleigh number $R_{\mathrm{t}}$, and the Lewis number Le. We examine the effects of these parameters while consider the fluid having constant viscosity, i.e. $\gamma=0$. On the 
other hand, we also consider the variable viscosity effect on the induced flow, which turns out to be crucial and complicated. The solution of the induced flow is shown in Eqs. (3.18) and (3.19), based on which the following discussion is made.

\subsection{Effective Taylor number effect}

The induced flow is affected by three physical parameters accounting for the inclined rotation: the inclined angle, the rotation speed, and the sense of rotation. As indicated by Chung and Chen [11], a larger inclined angle leads to a larger induced flow, a higher rotation speed induces a flow of smaller velocity, and the senses of spin and precession modify the induced flow. The effective Taylor number defined in Eq. (3.12) is able to account for the combined effect of these three physical parameters. According to Eq. (3.12), a larger $T_{\mathrm{e}}$ does not necessarily imply either larger spin speed or larger precession speed, depending on the relative sense between spin and precession and the inclination angle (accounted for by $C_{\mathrm{n}}$ ) as well. For example, as both spin and precession are of the same sense, i.e. $n_{\mathrm{p}}=n_{\mathrm{s}}=0$, a larger $T_{\mathrm{e}}$ means a smaller inclined angle and/or higher spin speed (accounted for by $\mathrm{Ta}_{\mathrm{s}}$ ) and precession speed (by $T a_{p}$ ). As spin and precession are of different sense of rotation, for example $n_{\mathrm{p}}=1, n_{\mathrm{s}}=0$, the relation among $T_{\mathrm{e}}, C_{\mathrm{n}}, \mathrm{Ta}_{\mathrm{p}}$ and $\mathrm{Ta}_{\mathrm{s}}$ is rather mixed, no general trend can be followed with. We shall therefore consider the first case, i.e. $n_{\mathrm{p}}=n_{\mathrm{s}}=0$, to interpret the results regarding the effect of $T_{\mathrm{e}}$.

A typical result is shown in Fig. 3(a), showing that the induced flow velocity decreases as $T_{\mathrm{e}}$ increases, or equivalently the induced flow is inhibited by a higher rotation speed through the action of Coriolis force. In other words, because the Coriolis force balances with the induced flow and rotation, the induced velocity must accordingly decrease as the rotation speed increases (larger $T_{\mathrm{e}}$ ). On the other hand, a larger inclination angle (a smaller $C_{\mathrm{n}}$ and a smaller $T_{\mathrm{e}}$ ) leads to a larger induced flow because the buoyancy component along the melt/solid interface increases. We note that the induced flow is also seen in the case as $T_{\mathrm{e}} \rightarrow 0$, implying clearly that the flow is induced by inclination, not by rotation. The $T_{\mathrm{e}}$ effect on the direction of the induced flow is constrained in the shallow layer above the interface (Fig. 3(b)) because its influence is significant only on the Ekman-layer flow, while is negligible on the
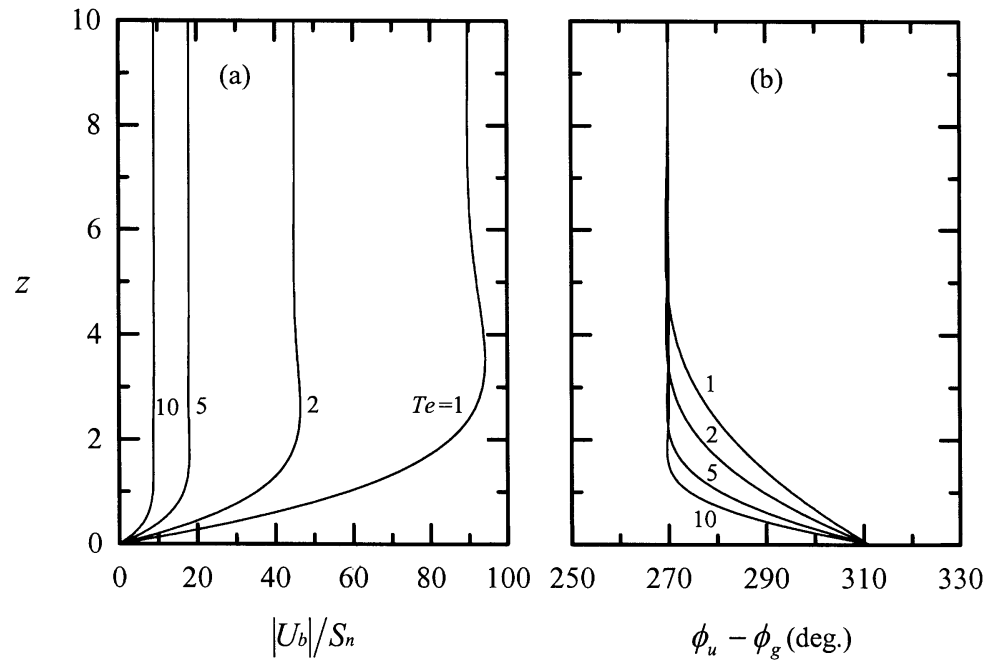

Fig. 3. The effective Taylor number $\left(T_{\mathrm{e}}\right)$ effect on the induced flow. A higher $T_{\mathrm{e}}$ leads to an induced flow of smaller velocity amplitude. (a) The velocity amplitude (b) the phase angle. 

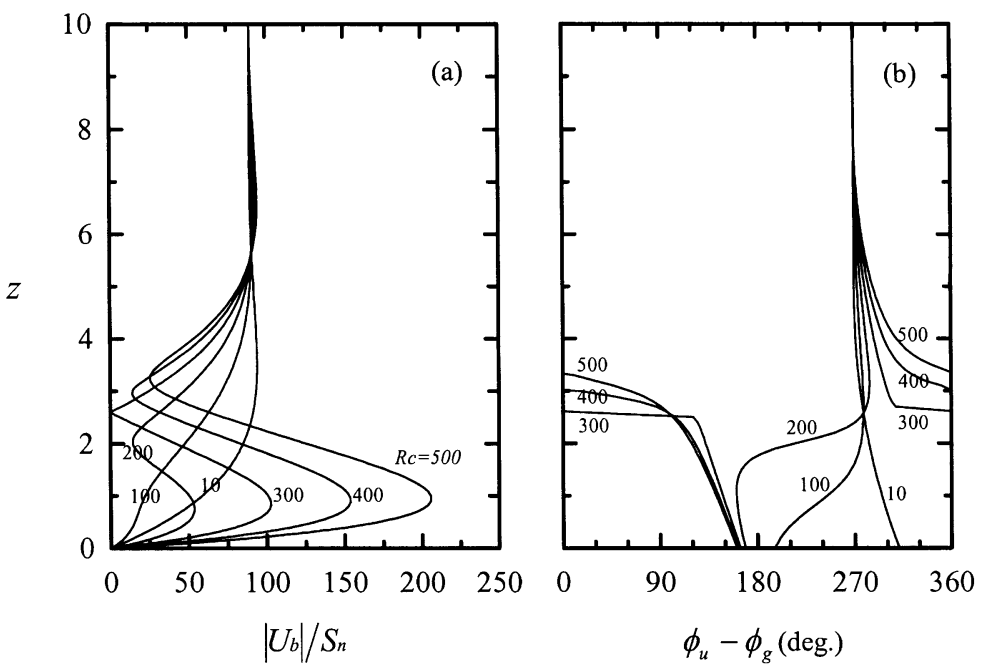

Fig. 4. The effect due to solutal Rayleigh number $\left(R_{\mathrm{c}}\right)$ variation, which contributes more significantly on the change of phase angle than the amplitude. (a) The velocity amplitude (b) the phase angle.

solutal-layer flow and is of virtually no influence on the thermal-layer flow.

\subsection{Solutal Rayleigh number effect}

The effect of solutal Rayleigh number is shown in Fig. 4 for the case $T_{\mathrm{e}}=1, R_{\mathrm{t}}=250$ and $\mathrm{Le}=$ 3600. It is known that solutal Rayleigh number generally accounts for the density gradient due to solute variation in $z$-direction and thus the buoyancy to drive convection. Because of the solutal gradient, a change of $R_{\mathrm{c}}$ will influence both the solutal-layer and the Ekman-layer flows because of the interaction between Coriolis force and the buoyancy. As a result, the influence of $R_{\mathrm{c}}$ is confined within the region in which the spiral-flow structure prevails while it has virtually no influence on the thermal-layer flow which dominates the induced flow beyond the spiral-region. The results of Fig. 4 show that, as $R_{\mathrm{c}}$ is small $(=10-100)$, its effect on either the solutal-layer flow or the Ekman-layer flow is negligible so that the induced flow is dominated by the thermal-layer flow. As $R_{\mathrm{c}}$ is large enough, its effect on both solutal-layer flow and Ekman-layer flow prevails, resulting in a dramatic change of velocity profile (in both amplitude and direction) within the spiral-region.
By and large, the induced flow increases dramatically the velocity within the shallow region above the interface and then decreases dramatically within another shallow region, and finally increases again to approach the thermal-layer flow in the outer region of the melt. On the other hand, an increase of $R_{\mathrm{c}}$ leads to an increase of velocity gradient above the interface, which may in turn influence the stability of morphology of the interface. It is therefore an important factor for precise control during the solidification process.

\subsection{Thermal Rayleigh number effect}

The thermal Rayleigh number $R_{\mathrm{t}}$ accounts for the density gradient due to temperature variation, which is a stabilizing factor for convective flow while it is a driving force to the induced flow when interacting with the Coriolis force. Accordingly, a change of $R_{\mathrm{t}}$ influences both the thermal-layer and Ekman-layer flows, as shown in Fig. 5 in which the case $T_{\mathrm{e}}=1, R_{\mathrm{c}}=10$ and $\mathrm{Le}=3600$ is considered. As $R_{\mathrm{t}}$ increases, both the thermal-layer and Ekman-layer flows increase their velocity amplitude, and as a result, the induced flow becomes more intensive (Fig. 5(a)). On the other hand, $R_{\mathrm{t}}$ has insignificant influence on the direction of 

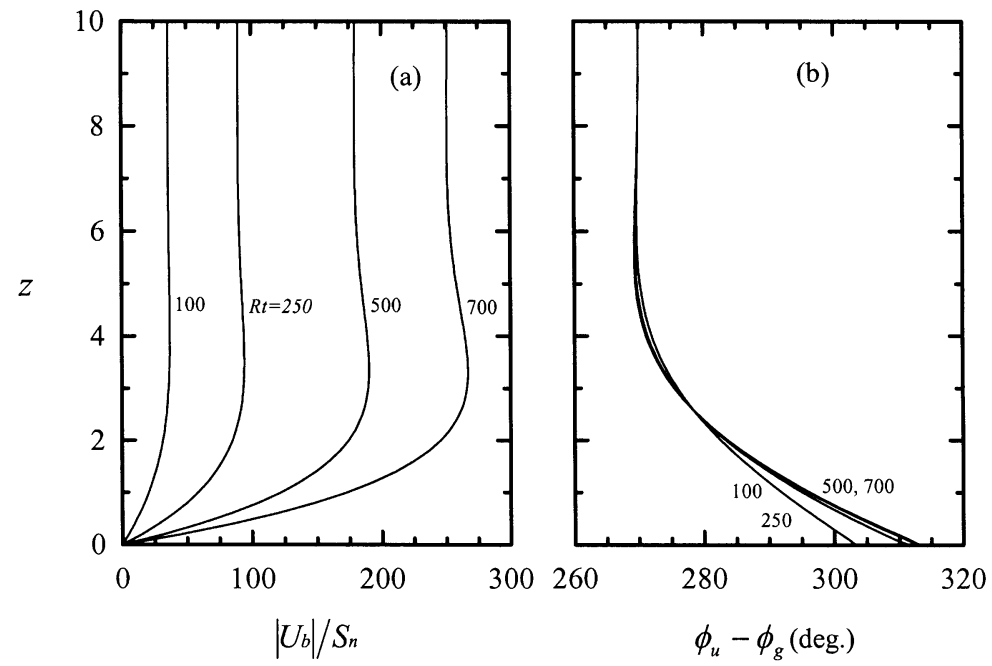

Fig. 5. The effect due to thermal Rayleigh number $\left(R_{\mathrm{t}}\right)$ variation, which contributes mainly on the velocity amplitude while has no influence on the phase angle. In general, a larger $R_{\mathrm{t}}$ results in a larger induced flow. (a) The velocity amplitude (b) the phase angle.

induced flow (Fig. 5(b)), because most of the $R_{\mathrm{t}}$ effect is reflected through the change of thermallayer flow, which does not change the direction in the melt.

\subsection{Lewis number effect}

Lewis number Le accounts for the ratio of thermal diffusivity to solutal diffusivity, or equivalently for the relative thickness of thermal boundary layer to solutal boundary layer above the interface. Namely, a larger Le means a thicker thermal boundary layer above the interface in comparison with the solutal boundary layer. Accordingly, as Le increases, the thermal gradient interacts with the Coriolis force in a thicker layer above the interface, resulting in a more intense induced flow (Fig. 6(a)). As Le decreases, on the other hand, the induced flow is less intense and the change of direction of the flow within the spiralregion is more obvious (Fig. 6(b)) because the solutal-gradient is becoming active in a thicker solutal boundary layer.

\subsection{The induced flow of variable viscosity}

To investigate the variable-viscosity effect on the induced flow, we consider the viscosity variation ranging from $\gamma=0$ to 10 , which accounts for an $\mathrm{e}^{10}$-fold (or a more than 20000-fold) change of viscosity due to temperature variation. The analytical solution of the induced flow is shown in Eqs. (3.18) and (3.19) and some typical results are shown in Fig. 7 for the case $R_{\mathrm{t}}=250$ and $T_{\mathrm{e}}=1$ when $R_{\mathrm{c}}$, Le and $\gamma$ vary. Results of Fig. 7 show that the influence of variable-viscosity, accounted for by changing $\gamma$, varies for different $R_{\mathrm{c}}$ and Le. As Le is small, see Figs. 7(a), (d) and (g), a small $R_{\mathrm{c}}(=10)$ leads to a larger viscosity in a larger region above the interface, resulting in that the induced flow is retarded by the large viscosity in a larger region. As $R_{\mathrm{c}}$ increases, the induced flow is confined to the shallower region above the interface because of the increasing prevailing of the interaction between buoyancy and Coriolis force within this region. The induced flow is virtually vanished when $\gamma$ is large, implying that the importance of viscosity-variation predominates when $R_{\mathrm{c}}$ is large.

As Le becomes large, the importance of viscosity-variation accounted for by varying $\gamma$ decreases, as shown by Figs. 7(b), (e) and (h). This is obviously due to the fact that the change of viscosity in this case has little effect on the induced flow, which can be seen also from Figs. 7(c), (f) and (i) where the effect of viscosity variation is 

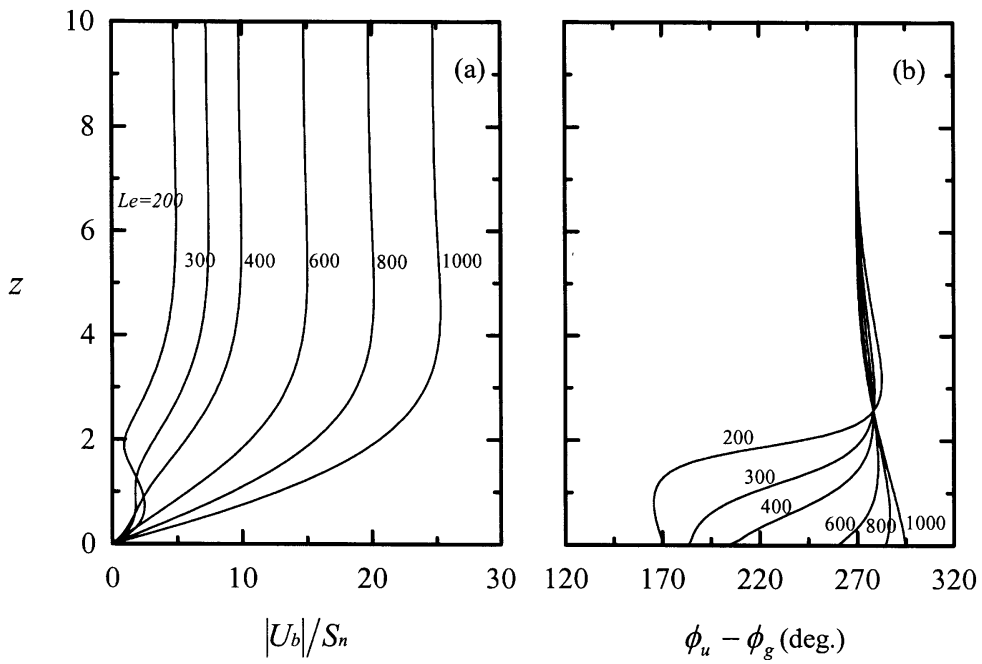

Fig. 6. The effect due to Lewis number (Le) variation. A larger Le leads to a larger induced flow. (a) The velocity amplitude (b) the phase angle.

very small. A larger Le implies a thicker thermal boundary layer, so that the viscosity contrast across the boundary layer is smaller than the case of smaller Le provided that the temperature difference across thermal boundary layer is the same. Consequently, as Le is large enough (such as 3600 ), the viscosity contrast across thermal boundary layer is relatively small, and therefore the influence due to temperature-dependent viscosity on the induced flow is also small.

\section{Concluding remarks}

We have investigated analytically the flow in the directional-solidifying melt under inclined rotation including spin and precession. Results show that the flow is driven by the gravity component along the melt/solid interface and is modified by the rotation including spin and precession through the interaction between the Coriolis force and the density gradient. The induced flow moves parallel to the interface while it changes the direction along the direction perpendicular to the interface, forming a spiral structure like an Ekman flow. Neither of the temperature nor the concentration distribution is influenced by the formation of induced flow. The induced flow consists of three compo- nents: the solutal layer flow, the thermal layer flow, and the Ekman layer flow; the third component dominates the induced flow by changing the flow direction periodically along vertical axis. The induced flow is influenced by the following physical parameters:

(a) The effective Taylor number $\left(T_{\mathrm{e}}\right)$ : The induced flow increases its velocity when inclined angle is larger, decreases when rotation speed is larger, and is modified by the senses of spin and precession. The effective Taylor number defined in Eq. (3.12) is able to account for the combined effect of these three physical factors, leading to the fact that a smaller $T_{\mathrm{e}}$ results in a more intensive induced flow.

(b) The solutal Rayleigh number $\left(R_{\mathrm{c}}\right)$ : an increase of $R_{\mathrm{c}}$ leads to an increase of velocity gradient above the melt/solid interface, which may in turn change the morphology of the interface and the quality of the final casting. It is therefore an important factor to the manufacture of high quality casting via a precise control on the solidification process.

(c) The thermal Rayleigh number $\left(R_{\mathrm{t}}\right)$ : As $R_{\mathrm{t}}$ increases, the induced flow becomes more intensive because, through the interaction with the Coriolis force, the thermal gradient 

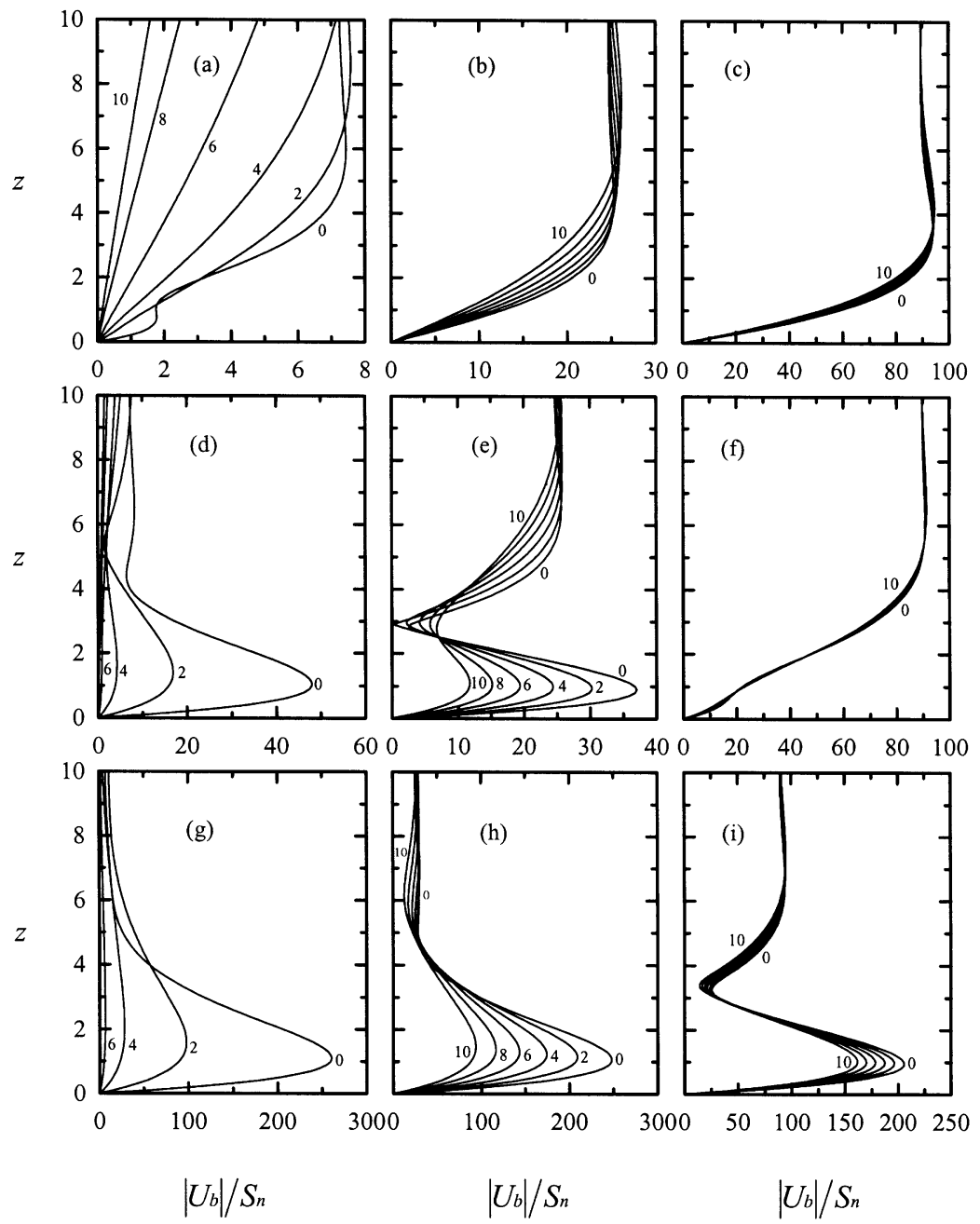

Fig. 7. The effect of viscosity variation for different $R_{\mathrm{c}}$ and Le. (a) $R_{\mathrm{c}}=10$, Le $=300$. (b) $R_{\mathrm{c}}=10$, Le $=1000$. (c) $R_{\mathrm{c}}=10$, Le $=3600$. (d) $R_{\mathrm{c}}=100, \mathrm{Le}=300$. (e) $R_{\mathrm{c}}=100, \mathrm{Le}=1000$. (f) $R_{\mathrm{c}}=100, \mathrm{Le}=3600$. (g) $R_{\mathrm{c}}=500, \mathrm{Le}=300$. (h) $R_{\mathrm{c}}=500$, Le $=1000$. (i) $R_{\mathrm{c}}=$ $500, \mathrm{Le}=3600$.

is a driving force to the formation of induced flow.

(d) The Lewis number (Le): A larger Le means a thicker thermal boundary layer above the interface in comparison with the solutal boundary layer. Accordingly, as Le increases, the thermal gradient interacts with the Coriolis force in a thicker layer above the interface, resulting in a more intense induced flow. (e) The temperature-dependent viscosity: The effect due to viscosity variation is smaller when either $R_{\mathrm{c}}$ or Le becomes larger. This is because, in general, the thickness of the thermal boundary layer plays a crucial role in determining the intensity of induced flow: a larger viscosity contrast across the thermal boundary layer leads to a more significant influence on the induced flow. 


\section{Acknowledgements}

The financial support to this research by National Science Council through NSC 89-2212E-002-124 is gratefully acknowledged.

\section{Appendix A}

The coefficients of Eqs. (3.18a)-(3.18c) are shown as follows:

$$
\begin{aligned}
& A_{1}=-\frac{S_{\mathrm{n}} R_{\mathrm{c}} G_{\mathrm{c}}}{\Delta c} \mathrm{e}^{i \phi_{\mathrm{c}}} \\
& A_{2}=-\frac{S_{\mathrm{n}} R_{\mathrm{t}} \mathrm{Le} G_{1}}{\Delta t} \mathrm{e}^{i \phi_{\mathrm{t}}} \\
& B_{1}=\frac{Q B A_{1}}{\Delta c} \mathrm{e}^{i \phi_{\mathrm{c}}} \\
& B_{2}=\frac{(2 Q-1 / \mathrm{Sc}) B_{1}}{\Delta c} \mathrm{e}^{i \phi_{\mathrm{c}}}-B_{1} \\
& B_{3}=\frac{Q B A_{2}}{\mathrm{Le} \Delta_{t}} \mathrm{e}^{i \phi_{\mathrm{t}}}, \\
& B_{4}=\frac{(2 Q / \mathrm{Le}-1 / \mathrm{Sc}) B_{3}}{\Delta t} \mathrm{e}^{i \phi_{\mathrm{t}}}-\mathrm{Le} B_{3} \\
& B_{5}=-Q B\left(A_{1}+A_{2}\right) a^{2} /(4 Q a+2 / \mathrm{Sc}) \\
& B_{6}=-\left[Q B\left(A_{1}+A_{2}\right) a+2 Q B_{5}\right] /(2 a Q+1 / \mathrm{Sc}) \\
& C_{1}=\frac{Q B\left[B_{1}-(1+B) A_{1} / 2\right]}{\Delta c} \mathrm{e}^{i \phi_{\mathrm{c}}}, \\
& C_{2}= \\
& \frac{Q B\left[B_{2}-3 B_{1}+(1+B) A_{1}\right]+(4 Q-2 / \mathrm{Sc}) C_{1}}{\Delta c} \mathrm{e}^{i \phi_{\mathrm{c}}}
\end{aligned}
$$$$
C_{3}=\frac{Q B\left(B_{1}-B_{2}\right)+(2 Q-1 / \mathrm{Sc}) C_{2}-2 Q C_{1}}{\Delta c} \mathrm{e}^{i \phi_{\mathrm{c}}},
$$

$$
C_{4}=\frac{Q B\left[B_{3}-(1+B) A_{2} / 2\right]}{\operatorname{Le}^{2} \Delta t} \mathrm{e}^{i \phi_{\mathrm{t}}},
$$

$$
\begin{aligned}
& C_{5}= \\
& \frac{Q B[}{\left.B_{4} / \mathrm{Le}^{2}-3 B_{3} / \mathrm{Le}+(1+B) A_{2} / \mathrm{Le}\right]+(4 Q / \mathrm{Le}-2 / \mathrm{Sc}) C_{4}} \\
& \times \mathrm{e}^{i \phi_{\mathrm{t}}}, \\
& C_{6}= \\
&\left.\frac{Q B(}{\Delta t} B_{3}-B_{4} / \mathrm{Le}\right)-2 Q C_{4}+(2 Q / \mathrm{Le}-1 / \mathrm{Sc}) C_{5} \\
& \Delta t \\
& \times \mathrm{e}^{i \phi_{\mathrm{t}}}, \\
& C_{7}= Q B a^{2} B_{5} /(8 Q a+4 / \mathrm{Sc}), \\
& C_{8}=\left\{Q B \left[a^{2} B_{6}+5 a B_{5}\right.\right. \\
&\left.\left.+(1+B)\left(A_{1}+A_{2}\right) a^{2} / 2\right]-12 Q C_{7}\right\} / \\
&(6 Q a+3 / \mathrm{Sc}) \\
& C_{9}=\left\{Q B \left[3 a B_{6}+4 B_{5}-\left(B_{2}+B_{4}\right) a^{2}\right.\right. \\
&\left.\left.+(1+B)\left(A_{1}+A_{2}\right) a\right]-6 Q C_{8}\right\} / \\
&(4 Q a+2 / \mathrm{Sc}), \\
& C_{10}=\left\{Q B\left[B_{6}-\left(B_{2}+B_{4}\right) a\right]-2 Q C_{9}\right\} / \\
&(2 Q a+1 / \mathrm{Sc}),
\end{aligned}
$$

where

$$
\begin{aligned}
& \Delta c=\left[(Q-1 / \mathrm{Sc})^{2}+T_{\mathrm{e}}^{2}\right]^{1 / 2}, \\
& \cos \phi_{\mathrm{c}}=\frac{Q-1 / \mathrm{Sc}}{\Delta c}, \\
& \sin \phi_{\mathrm{c}}=\frac{T_{\mathrm{e}}}{\Delta c}, \\
& \Delta t=\left[\left(Q \mathrm{Le}^{-2}-\mathrm{Sc}^{-1} \mathrm{Le}^{-1}\right)^{2}+T_{\mathrm{e}}^{2}\right]^{1 / 2}, \\
& \cos \phi_{\mathrm{t}}=\frac{\left[Q \mathrm{Le}^{-2}-(\mathrm{ScLe})^{-1}\right]}{\Delta t}, \\
& \sin \phi_{\mathrm{t}}=\frac{T_{\mathrm{e}}}{\Delta t} .
\end{aligned}
$$

\section{References}

[1] J.W. Rutter, B.A. Chalmers, Can J. Phys. 31 (1953) 15.

[2] S.R. Coriell, M.R. Cordes, W.J. Boettinger, R.F. Sekerka, J. Crystal Growth 49 (1980) 13.

[3] R.T. Delves, J. Crystal Growth 3 (1968) 562.

[4] R.T. Delves, J. Crystal Growth 8 (1971) 13.

[5] S.R. Coriell, G.B. McFadden, R.F. Boisvert, R.F. Sekerka, J. Crystal Growth 69 (1984) 15. 
[6] G.B. McFadden, S.R. Coriell, J.I.D. Alexander, Commun. Pure Appl. Math. 41 (1988) 683.

[7] S.A. Forth, A.A. Wheeler, J. Fluid Mech. 202 (1989) 339.

[8] S.A. Forth, A.A. Wheeler, J. Fluid Mech. 236 (1992) 61.

[9] C.A. Chung, F. Chen, J. Fluid Mech. 436 (2001) 85.

[10] A.K. Sample, A. Hellawell, Metall. Trans. A15 (1984) 2163.
[11] C.A. Chung, F. Chen, J. Fluid Mech. 412 (2000) 93.

[12] D. Turnbull, Trans. Met. Soc. AIME 212 (1961) 422.

[13] H.R. Thresh, A.F. Crawley, Met. Trans. 1 (1970) 1531.

[14] K.C. Steingel, D.S. Oliver, J.R. Booker, J. Fluid Mech. 120 (1982) 411.

[15] S.H. Davis, J. Fluid Mech. 212 (1990) 242. 